\title{
Musulju improves benign prostatic hyperplasia by regulating inflammatory and apoptotic proteins
}

\author{
JAE-YUN JU, KYUNG-SOOK CHUNG, SE-YUN CHEON and HYO-JIN AN \\ Department of Pharmacology, College of Korean Medicine, Sangji University, \\ Wonju-si, Gangwon-do 220-702, Republic of Korea
}

Received September 28, 2015; Accepted September 23, 2016

DOI: $10.3892 / \mathrm{mmr} .2016 .5839$

\begin{abstract}
Alternative medicine is a widely accepted therapeutic approach for the management of various diseases. The Korean medicine, musulju (MSJ), has been traditionally used to improve vital energy in men with reduced physical strength and a weakened urinary system. The present study determined the mechanisms underlying the protective effect of MSJ against benign prostatic hyperplasia (BPH), a common disorder in elderly men that involves inflammation-mediated imbalance between cell proliferation and death. MSJ treatment was demonstrated to decrease prostate weight, cell proliferation, and the protein expression of proliferating cell nuclear antigen in a rat model of BPH. In addition, MSJ markedly reduced serum testosterone levels, $5 \alpha$-reductase 2 mRNA expression and $\mathrm{BPH}$-associated upregulation of inflammatory proteins, inducible nitric oxide synthase and cyclooxygenase 2 . Furthermore, MSJ induced apoptosis by regulating B-cell lymphoma $(\mathrm{Bcl})-2$ protein expression and the $\mathrm{Bcl}-2$ :Bax ratio, leading to caspase 3 activation. Taken together, MSJ demonstrated antiproliferative effects in BPH model rats by regulating the expression levels of proteins involved in inflammation and apoptosis. The effects of MSJ may be attributed to its alternative therapeutic properties.
\end{abstract}

\section{Introduction}

Benign prostatic hyperplasia (BPH), also commonly termed benign prostatic hypertrophy, can be described clinically or pathologically. Clinical BPH presents as benign enlargement of the prostate, which contributes to an array of voiding difficulties that can range from bothersome to significantly impacting quality of life in older men (1). Due to the high prevalence

Correspondence to: Professor Hyo-Jin An, Department of Pharmacology, College of Korean Medicine, Sangji University, 83 Sangjidae-gil, Wonju-si, Gangwon-do 220-702, Republic of Korea

E-mail: hjan@sj.ac.kr

Key words: musulju, benign prostatic hyperplasia, testosterone, inflammation, apoptosis of BPH in elderly men, BPH has been suggested to be a ubiquitous sign of aging (2). The concept that androgens are important for the maintenance of prostate disease dictates the standard of care for BPH (3). Although aging and androgens are two established factors that contribute to the development of $\mathrm{BPH}$, recent novel findings highlight the importance of inflammation (4). Upon histological examination, almost all BPH specimens exhibit inflammatory infiltrates; however, the link to bacterial or other foreign antigens remains to be elucidated. Auto-reactive T cells recognize prostate secretion products and animal models of experimental prostatitis have demonstrated an autoimmune component of chronic inflammation (5). Therefore, reducing inflammation may serve a crucial role in the treatment of BPH and may lead to improved clinical outcomes. Various types of pharmaceutical therapies are in use for the treatment of $\mathrm{BPH}$, including alternative herbal-based therapies (6). Typical herbal therapies recommended for BPH with certain clinical evidence of efficacy include saw palmetto (Serenoa repens), stinging nettle (Urtica dioica) and Pygeum africanum (7). Bee pollen extract (cernilton) has also been used; however, less evidence of its efficacy against BPH exists. Lower urinary tract symptoms provide a complex but common connection between $\mathrm{BPH}$ and chronic prostatitis. Therefore, alternative agents can be used alone or in combination for treatment of $\mathrm{BPH}$.

The herbal liquor, musulju (MSJ), has been traditionally used in Korean medicine to strengthen virility. The Korean medicine book called the Dongui Bogam reported MSJ to be effective in elderly men for alleviating urinary system dysfunction. Although MSJ may be a potential therapeutic for $\mathrm{BPH}$, the molecular mechanisms supporting its clinical claims remain elusive. Therefore, to understand the mechanisms underlying its clinical effect, the present study evaluated the anti-proliferative effect of MSJ and determined the molecular mechanisms of MSJ in a testosterone-induced rat model of $\mathrm{BPH}$.

\section{Materials and methods}

Chemicals and reagents. Testosterone, phenylmethylsulfonyl fluoride, Triton-X-100 and the protein inhibitor cocktail were purchased from Sigma-Aldrich (St. Louis, MO, USA). Finasteride, a type II 5-reductase inhibitor, was obtained from Merck \& Co., Inc. (Whitehouse Station, NJ, USA). $5 \alpha$-Reductase 2 and 
glyceraldehyde-3-phosphate dehydrogenase $(G A P D H)$ oligonucleotide primers were purchased from Bioneer Corporation (Daejeon, Republic of Korea), and SYBR Premix Ex Taq was purchased from Takara Bio., Inc. (Otsu, Japan). Antibodies against inducible nitric oxide synthase (iNOS; M-19; cat. no. sc-650), cyclooxygenase 2 (COX-2; C-20; cat. no. sc-1745), poly (ADP-ribose) polymerase-1 (PARP-1; F-2; cat. no. sc-25780), caspase-3 (E-8; cat. no. sc-7272), Bcl-2 (C-2; cat. no. sc-7382), Bcl-xL (H-5; cat. no. sc-8392), Bax (B-9; cat. no. sc-7480) and $\beta$-actin (ACTBD11B7; cat. no. sc-81178) were purchased from Santa Cruz Biotechnology, Inc. (Dallas, TX, USA). An antibody for proliferating cell nuclear antigen (PCNA; cat. no. sc-56) was purchased from BD Biosciences (San Jose, CA, USA). Horseradish peroxidase-conjugated secondary antibody was purchased from Jackson ImmunoResearch Laboratories, Inc. (West Grove, PA, USA).

Preparation of MSJ. The muscle of Canis familiaris L. (1.5 kg) was purchased from the Moran traditional market, (Sung-nam, Korea), and it was cleansed. It was boiled for $24 \mathrm{~h}$ and the oil, which forms on the top, was removed. It was subsequently mixed with hard-steamed glutinous rice $(24 \mathrm{~kg})$ and malt made of barley flour (120 g), and it was brewed for 14 days. MSJ was filtered through Whatman No. 1 filter paper and the filtrate was evaporated to dryness under reduced pressure on an EYELAN-1000 rotary evaporator (EYELA Riakikai Co., Ltd, Tokyo, Japan) at $40^{\circ} \mathrm{C}$. Finally, MSJ extracts were obtained and stored at $-20^{\circ} \mathrm{C}$ for later use.

Animals. Male Wistar rats $(\mathrm{n}=24 ; 10$-weeks-old; weight, $200 \pm 20 \mathrm{~g}$ ) were purchased from Daehan Biolink (Daejeon, Korea). The animals were housed under conditions specified in the guide for the Care and Use of Laboratory Animals, as adopted and promulgated by the Institutional Animal Care Committee, Sangji University (Reg. no. 2014-08; Wonju-si, Korea). Prior to the start of the experiment, the rats were adapted to the modified conditions for 2 weeks. Rats were randomly distributed into four groups (n=6/group): i) Sham-operated group (Con); ii) BPH model group (BPH); iii) finasteride-administrated group (Fina); and iv) Musulju-treated group (MSJ). The rats were castrated, with the exception of those in the Con group, and BPH was induced in castrated animals by subcutaneous injection of testosterone, as described previously (8). Throughout the experiment, the animals were provided free access to food and water for 4 weeks, and they were maintained under a $12 \mathrm{~h}$ light/dark cycle at a constant temperature of $22 \pm 2^{\circ} \mathrm{C}$ with a relative humidity of $55 \pm 9 \%$. At the end of the 4 -week period, the body weight of the rats was recorded and all animals were fasted for $12 \mathrm{~h}$. The following day, the rats were sacrificed by anesthesis with Zoletil ${ }^{\circledR} 50$ (intraperitoneal, $20 \mathrm{mg} / \mathrm{kg}$; Virbac, CarrosCedex, France), and blood samples were collected by cardiac puncture. The prostatic tissue was excised, rinsed, weighed and stored at $-70^{\circ} \mathrm{C}$ until further analysis.

Administration and dosage. Testosterone propionate (Wako Pure Chemical Industries, Ltd., Osaka, Japan) was diluted with corn oil and injected subcutaneously $(10 \mathrm{mg} / \mathrm{kg})$ into rats in the BPH, Fina and MSJ groups to induce BPH $(9,10)$. Finasteride (Merck \& Co., Inc.) and MSJ were dissolved in distilled water. Fina and MSJ were orally administered at a dose of 5 and $200 \mathrm{mg} / \mathrm{kg}$, respectively.

Prostate weight to body weight ratio $(P W / B W)$. Prostatic tissues were excised, rinsed and weighed immediately after removal. The PW/BW ratio was calculated using the following equation: $\mathrm{PW} / \mathrm{BW}$ ratio $=$ (prostate weight of each animal from experimental group / body weight of each animal from experimental group) x 1,000 .

Growth inhibition of prostate weight. The percentage of growth inhibition was calculated as follows: 100 - [(treated group - control group) / (BPH group - control group) x 100].

Serum concentrations of testosterone analysis. The serum concentrations of testosterone were determined using commercial enzyme linked immunosorbent assay (ELISA) kits (Cayman Chemical Company, Ann Arbor, MI, USA). The assays were performed according to the manufacturer's protocol.

Histological analysis. The prostatic tissue in each group was fixed in $4 \%$ formalin, embedded in paraffin and the tissue was then cut into $4-\mu \mathrm{m}$ sections. The sections were stained with hematoxylin and eosin for histological examination. Images were captured using a SZX10 microscope (Olympus Corporation, Tokyo, Japan). The thickness of epithelium tissue from prostate (TETP) was measured using Leica Application Suite (ver.3.3.0) software (Leica Biosystems, Wetzlar, Germany) for histological analysis.

Reverse transcription-quantitative polymerase chain reaction $(R T-q P C R)$. The prostatic tissue from each animal was homogenized and the total RNA was isolated using Easy-Blue ${ }^{\circledR}$ reagent (iNtRON Biotechnology, Inc., Gyeonggi-do, Republic of Korea), according to the manufacturer's protocol. The total RNA was quantified using an Epoch ${ }^{\circledR}$ microvolume spectrophotometer system (BioTek Instruments, Inc., Winooski, VT, USA). Total RNA from the prostate was reverse transcribed into cDNA using a high-capacity cDNA reverse transcription kit (Life Technologies, Grand Island, NY, USA). PCR amplification was performed with the incorporation of SYBR green (Life Technologies; Thermo Fisher Scientific, Inc., Waltham, MA, USA). The oligonucleotide primers for designed from rat were as follows: $5 \alpha$-reductase 2, forward: 5'-ATG GGG ACC CTG ATC CTG TG-3' and reverse: 5'-CGA CAC CAC AAA GGA AGG CA-3'; GAPDH, a housekeeping gene, forward: 5'-TGA TTC TAC CCA CGG CAA GT-3' and 5'-AGC ATC ACC CCA TTT GAT GT-3'. RT was performed with a thermo cycler (Gene Amp ${ }^{\circledR}$ PCR system 9700; Life Technologies; Thermo Fisher Scientific, Inc.) and the results were expressed as the ratio of optimal density against that of GAPDH.

Western blot analysis. The prostatic tissue from each animal was homogenized in PRO-PREP ${ }^{\circledR}$ lysis buffer (iNtRON Biotechnology, Inc., Seoul, South Korea) and incubated for $25 \mathrm{~min}$ on ice to induce cell lysis. Tissue extracts were centrifuged at $13,000 \mathrm{rpm}\left(4^{\circ} \mathrm{C}\right)$ for $20 \mathrm{~min}$ and the supernatants were transferred to clean tubes. Aliquots of each protein sample $(30 \mu \mathrm{g})$ were resolved on $8-12 \%$ sodium dodecyl sulfate-polyacrylamide gel electrophoresis gels and were 
transferred onto a polyvinylidene fluoride membrane. The membranes were incubated for $1 \mathrm{~h}$ with blocking solution and were subsequently incubated with a 1:1,000 dilution of primary antibodies, including anti-PCNA, anti-iNOS, anti-COX-2, anti-PARP-1, anti-caspase-3, anti-Bcl-xL, anti-Bax, and anti- $\beta$-actin, overnight at $4^{\circ} \mathrm{C}$. The membranes were washed three times with $0.1 \%$ Tween-20/TBS, followed by incubation with the corresponding secondary antibodies $(1: 2,000$ dilution) for $1 \mathrm{~h}$ at room temperature. The membranes were washed as before, and the immunoreactive protein bands were visualized using enhanced chemiluminescence and exposure to X-ray film (GE Healthcare Bio-Sciences, Piscataway, NJ, USA). Density of protein band was measured using Bio-Rad Quantity One Software (version 4.6.3; Bio-Rad Laboratories, Inc., Hercules, CA, USA).

Statistical analysis. The data are expressed as the mean \pm standard error of the mean of 6 rats. The data were analyzed by one-way analysis of variance with Dunnett's test. Statistical analysis was performed using SPSS version 19.0 (IBM Corporation, Armonk, NY, USA).

\section{Results}

Effects of MSJ on prostate weight in BPH model rats. As shown in Fig. 1A, the mean prostate weight of rats in the BPH group was significantly higher compared with that of rats in the other groups, suggesting that testosterone successfully induced BPH in the castrated rats. The prostate weight of the rats in the Fina and MSJ groups significantly decreased compared with that of rats in the BPH group (Fig. 1A). The prostate weight in the BPH-induced group was 2.16-fold higher compared with that of the Con group. In the Fina and MSJ groups, the prostate weights were 1.25- and 1.14-fold higher, respectively, compared with the Con group. In addition, the $\mathrm{PW} / \mathrm{BW}$ ratio in $\mathrm{BPH}$ group was 2.31-fold higher compared with that in the Con group (Fig. 1B). The PW/BW ratios in the Fina and MSJ groups were 1.26- and 1.53-fold higher, respectively, compared with that in the Con group. In addition, the growth inhibition ratio of prostate growth in each group is demonstrated in Table I. In the Fina and MSJ groups, the prostate growth inhibition ratio was recorded to be almost $85 \%$ and $68 \%$, respectively.

Effects of MSJ on serum testosterone levels and $5 \alpha$-reductase $2 \mathrm{mRNA}$ expression in BPH model rats. Circulating testosterone acts locally in the prostate via production of growth factors that act in an autocrine or a paracrine manner to influence prostate cell growth, survival or apoptosis (11). As shown in Fig. 2A, serum testosterone levels in the testosterone-induced BPH group $(12.88 \pm 3.00 \mathrm{pg} / \mathrm{ml})$ were significantly higher compared with that in the Con group $(1.28 \pm 0.36 \mathrm{pg} / \mathrm{ml})$. However, serum testosterone levels in the Fina group $(8.70 \pm 0.00 \mathrm{pg} / \mathrm{ml})$ and the MSJ group $(5.93 \pm 0.51 \mathrm{pg} / \mathrm{ml})$ significantly decreased compared with that in the BPH group. In the prostate, testes and hair follicles, circulating testosterone is converted by $5 \alpha$-reductase to dihydrotestosterone (DHT), which serves a key role in the development of male reproductive organs, and these hormones are commonly associated with BPH (12). To determine if the effect of MSJ was associated with the synthesis of DHT from
Table I. Effect of treatments on prostate growth in rats treated with testosterone.

\begin{tabular}{lcc}
\hline Group & Prostate weight $(\mathrm{g})$ & Inhibition ratio (\%) \\
\hline Control & $0.87 \pm 0.20$ & - \\
BPH & $1.87 \pm 0.13^{\mathrm{a}}$ & - \\
Finasteride & $1.09 \pm 0.07^{\mathrm{b}}$ & 85.34 \\
MSJ & $0.99 \pm 0.39^{\mathrm{b}}$ & 68.26 \\
\hline
\end{tabular}

${ }^{\mathrm{a}} \mathrm{P}<0.001$ vs. Control group; ${ }^{\mathrm{b}} \mathrm{P}<0.001$ vs. $\mathrm{BPH}$ group. $\mathrm{BPH}$, benign prostatic hyperplasia; MSJ, musulju.

testosterone by $5 \alpha$-reductase, the present study examined the mRNA expression levels of $5 \alpha$-reductase 2 in prostatic tissue. As shown in Fig. 2B, similar to the results in the Fina group, MSJ treatment significantly decreased testosterone-induced mRNA expression of $5 \alpha$-reductase 2 in the prostate tissue compared with that in the BPH group.

Effects of MSJ on histological parameters and cell proliferation in BPH model rats. Histological analysis revealed changes in characteristics of glandular hyperplasia with epithelial proliferation and decreased glandular luminal area in the BPH model rats (Fig. 3A). However, Fina and MSJ treatment suppressed these typical hyperplastic patterns, which represent the histological change of normal prostatic tissue into tissue with prostatic hyperplasia. As shown in Fig. 3B, TETP analysis revealed that the thickness of the epithelium tissue was maximal in rats in the BPH group and that Fina and MSJ treatment significantly reduced the thickness of the epithelium tissue of the prostate.

In order to evaluate the effects of MSJ on the proliferation of prostatic epithelial cells, the present study examined the protein expression levels of PCNA, a proliferation marker, in the prostatic tissue of $\mathrm{BPH}$ model rats. As shown in Fig. 3C, PCNA protein levels, as detected by western blotting, increased in the $\mathrm{BPH}$ group relative to the levels in the Con group. Compared with the BPH group, however, the Fina and MSJ groups exhibited a slight increase in the protein levels of PCNA, consistent with the antiproliferative effects in BPH.

Effects of MSJ on inflammatory proteins in BPH model rats. Inflammatory factors serve a crucial role in proliferation of prostatic cells in BPH. As shown in Fig. 4, treatment with testosterone markedly increased the protein expression levels of iNOS and COX-2 in the BPH group compared with that of the control group. The Fina and MSJ groups, however, exhibited reduced expression levels of these inflammatory proteins.

Effects of MSJ on apoptotic proteins in BPH model rats. Apoptotic activity has been suggested to be a key cofactor in the development and progression of BPH. In the present study, treatment with testosterone upregulated the protein expression levels of PARP-1 and procaspase-3 in the BPH group (Fig. 5A). Treatment with MSJ, however, markedly suppressed the protein expression levels of PARP-1 and 

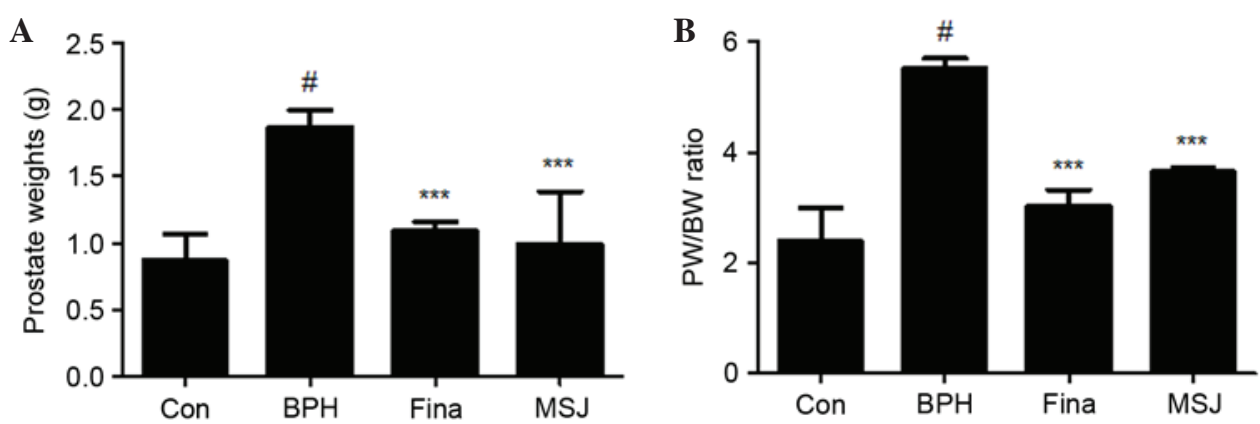

Figure 1. Effect of MSJ administration on prostate weight in BPH-induced rat models. Changes in (A) prostate total weight and (B) PW/BW ratio was assessed for the Con, BPH-induced, Fina and MSJ groups. The data are presented as the mean \pm standard error of the mean of 6 rats per group $\left({ }^{\#} \mathrm{P}<0.05\right.$ vs. Con group; ${ }^{* * *} \mathrm{P}<0.001$ vs. BPH group). BPH, benign prostatic hyperplasia; $\mathrm{PW} / \mathrm{BW}$, prostate weight/body weight ratio; Fina, BPH-induced group treated with finasteride $5 \mathrm{mg} / \mathrm{kg} / \mathrm{day}$; MSJ, BPH-induced group treated with musulju $200 \mathrm{mg} / \mathrm{kg} / \mathrm{day}$; Con, control.
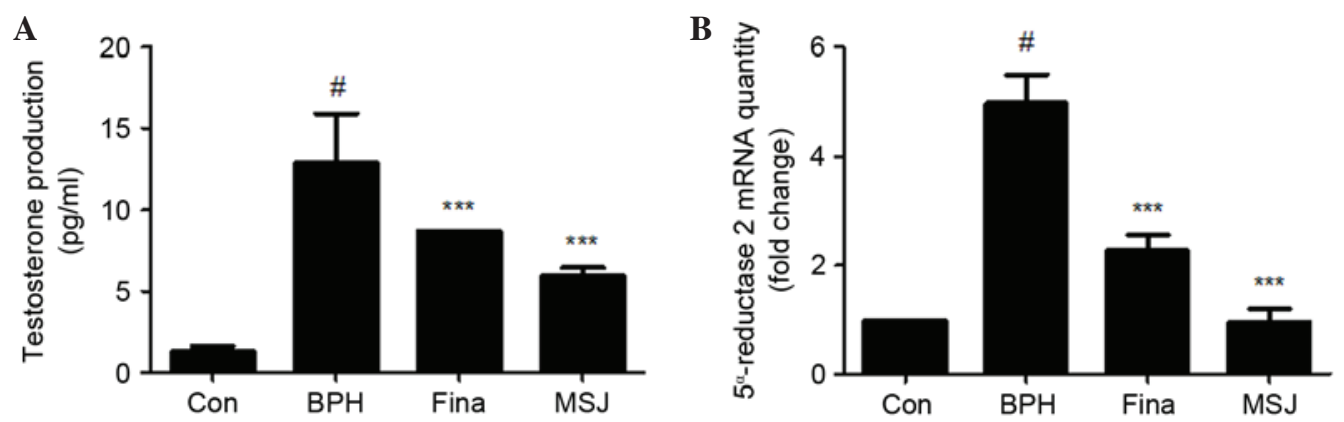

Figure 2. Effect of MSJ administration on the serum testosterone production and mRNA level of $5 \alpha$-reductase 2 in prostate tissues of BPH-induced rat models. (A) The serum concentrations of testosterone were determined using ELISA. (B) The mRNA expression of $5 \alpha$-reductase 2 in prostate tissue was analyzed by reverse transcription-quantitative polymerase chain reaction for the Con, BPH-induced, Fina and MSJ groups. The data are presented as the mean \pm standard error of the mean of 6 rats per group $\left({ }^{*} \mathrm{P}<0.05\right.$ vs. Con group; ${ }^{* * *} \mathrm{P}<0.001$ vs. BPH group). BPH, benign prostatic hyperplasia; Fina, BPH-induced group treated with finasteride $5 \mathrm{mg} / \mathrm{kg} /$ day; MSJ, BPH-induced group treated with musulju $200 \mathrm{mg} / \mathrm{kg} /$ day; Con, control.

procaspase-3, suggesting the involvement of caspase-mediated apoptotic pathways in BPH. In addition, the MSJ-treated group exhibited decreased levels of the antiapoptotic proteins, $\mathrm{Bcl}-2$ and Bcl-xL, but increased expression of proapoptotic Bax compared with the BPH group (Fig. 5B). Therefore, the ratio of Bcl-2 to Bax significantly decreased following treatment with MSJ, which suggested a role of the Bcl-2 family of proteins (Fig. 5C), an important mediator of the intrinsic apoptotic pathway, in MSJ-induced apoptosis in the BPH model. Therefore, MSJ-induced apoptosis was mediated by the regulation of the expression levels of the caspase and Bcl-2 family proteins.

\section{Discussion}

$\mathrm{BPH}$ is the most common benign proliferative disease in males, with $\sim 8$ million new patients diagnosed with primary or secondary BPH annually (13). BPH is the major cause of lower urinary tract symptoms, and is characterized by enlargement of the transitional zone and increased population of stromal cells (14). Numerous factors have been implicated in the pathogenesis of BPH, including an imbalance in cell proliferation and cell death (15). In the present study, the testosterone-induced BPH group demonstrated increased prostate weight and $\mathrm{PW} / \mathrm{BW}$ ratio compared with the Con group, and treatment of BPH model rats with $200 \mathrm{mg} / \mathrm{kg}$ MSJ orally for 4 weeks significantly inhibited this increase in prostate weight and $\mathrm{PW} / \mathrm{BW}$ ratio. In addition, histological analysis and assessment of epithelial thickness confirmed that MSJ was effective in reversing typical hyperplastic patterns, as previously shown. Based on these data, a dose of $200 \mathrm{mg} / \mathrm{kg}$ MSJ was efficacious in protecting against testosterone-induced BPH and this dose was used for further investigation.

Androgens serve an essential role in the development and growth of the entire male genital tract, in particular, the prostate, stimulating the differentiation and proliferation of both the epithelial and the stromal compartments of the gland (16). Development and maintenance of the normal prostate, as well as development of BPH, depend on a functional androgen-signaling axis, components that include: i) Testosterone synthesis in the testes and the adrenal glands; ii) conversion of testosterone to DHT by $5 \alpha$-reductase; iii) transport of DHT to target tissues; and iv) binding of DHT to the androgen receptor with consequent modulation of genes (17). 5 $\alpha$-reductase is responsible for the conversion of testosterone into its active form, DHT, and its activity is correlated with the occurrence and progression of BPH (18). In the present study, it was revealed that treatment with MSJ inhibited serum testosterone production in BPH model rats. Consistent with this reduction in serum testosterone levels, the mRNA expression of $5 \alpha$-reductase 2 was upregulated in rats with BPH, but was decreased in the animals receiving MSJ treatment. 

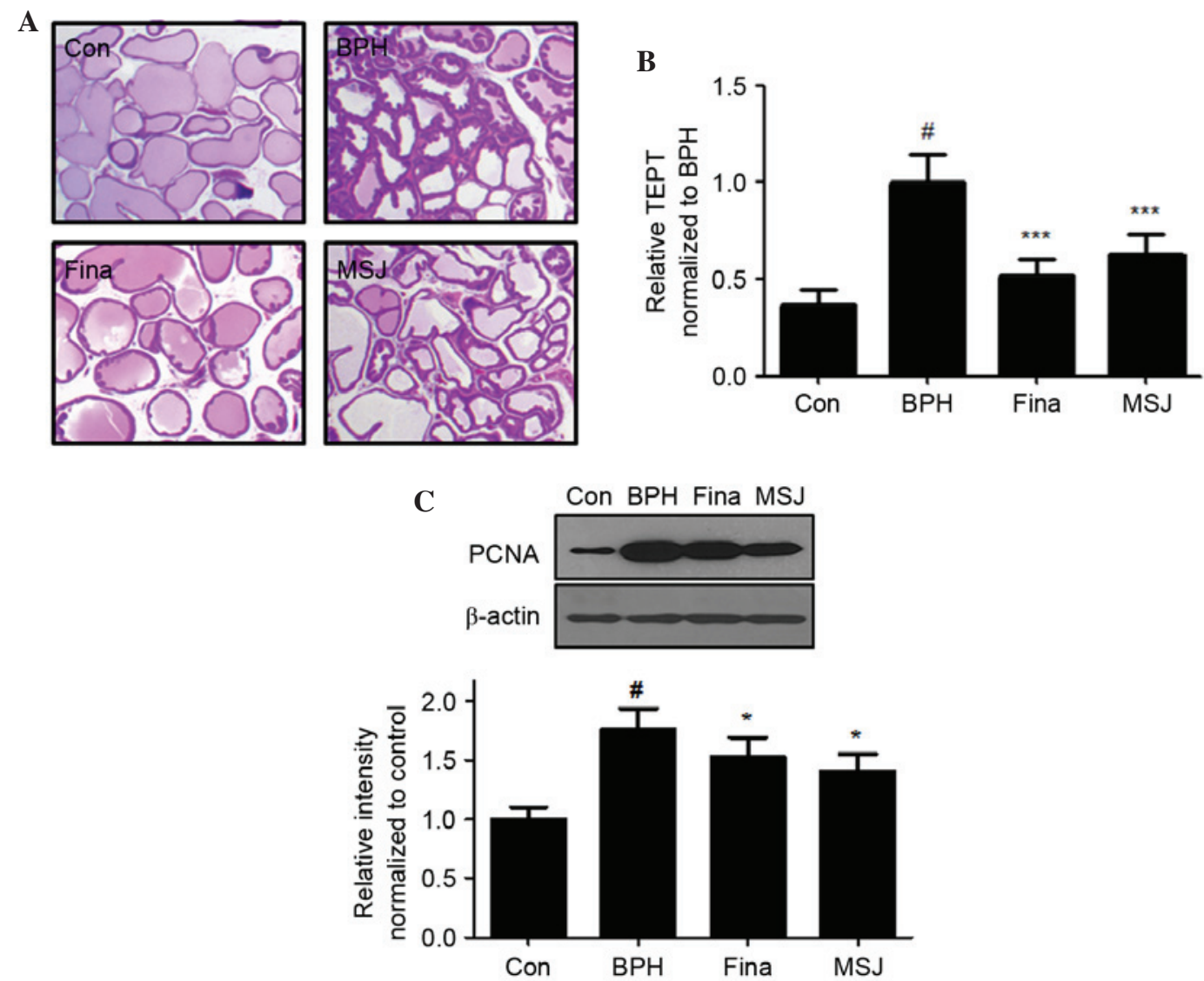

Figure 3. Effect of MSJ administration on the prostatic cell proliferation. (A) Hematoxylin and eosin staining of prostatic tissue from BPH-induced rat models was used to determine (B) the relative thickness of epithelium tissue from prostate TETP normalized against BPH (magnification, $\mathrm{x} 40$ ). (C) The protein expression of PCNA was determined by western blotting using specific antibodies. $\beta$-actin was used as an internal control. Densitometric analysis was performed using Bio-rad Quantity One ${ }^{\circledR}$ Software. The data are presented as the mean \pm standard error of the mean of 6 rats per group $\left({ }^{*} \mathrm{P}<0.05\right.$ vs. Con group; $\mathrm{P}<0.05$, ${ }^{* * * *} \mathrm{P}<0.001$ vs. BPH group). BPH, benign prostatic hyperplasia; Fina, BPH-induced group treated with finasteride $5 \mathrm{mg} / \mathrm{kg} / \mathrm{day}$; MSJ, BPH-induced group treated with musulju $200 \mathrm{mg} / \mathrm{kg} / \mathrm{day}$; Con, control; PCNA, proliferating cell nuclear antigen.
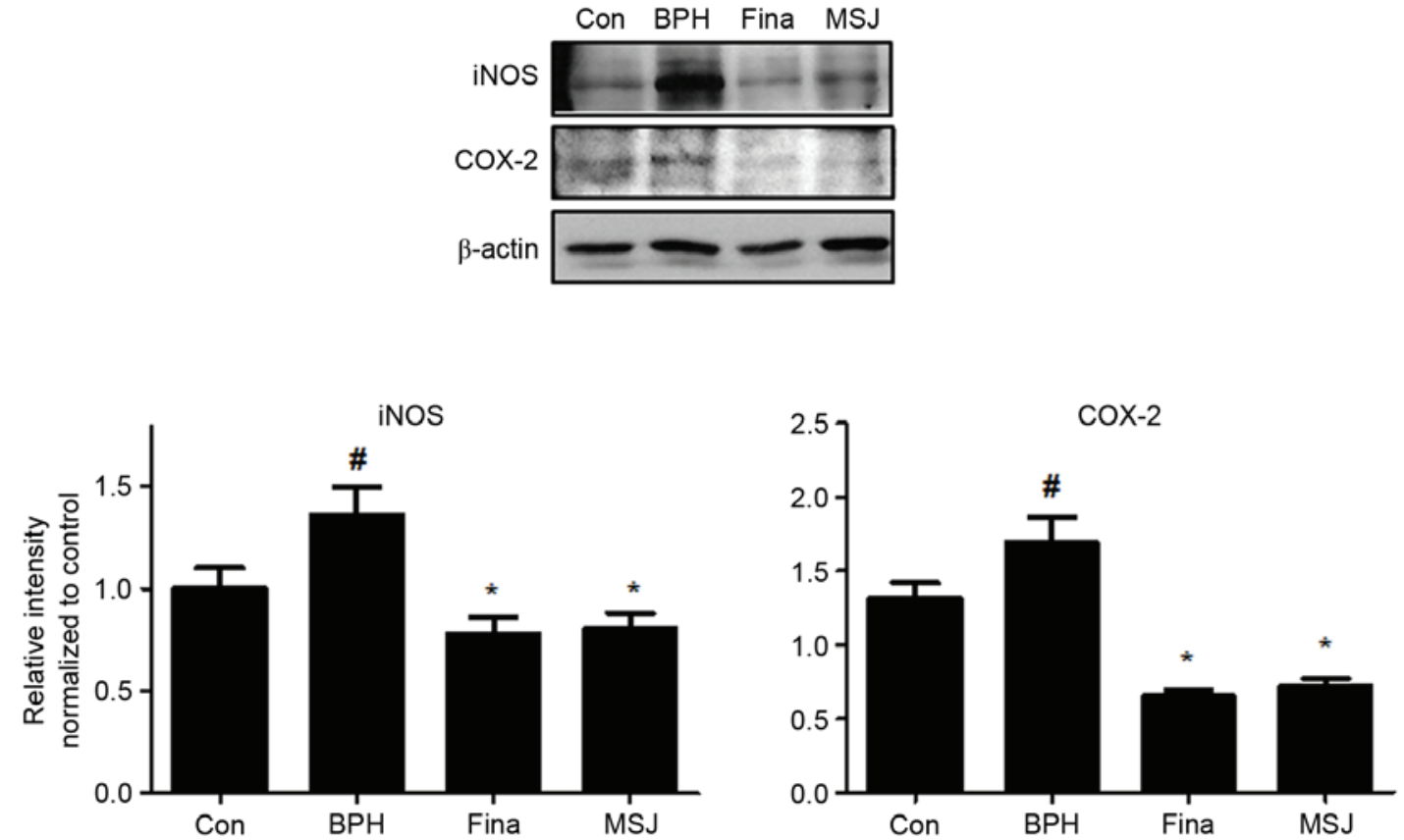

Figure 4. Effect of MSJ administration on the expression of iNOS and COX-2 in prostate tissues of BPH-induced rat models. The expression levels of iNOS proteins and COX-2 proteins were determined by western blotting using specific antibodies. $\beta$-actin was used as an internal controls. Densitometric analysis was performed using Bio-rad Quantity One ${ }^{\circledR}$ Software. The data presented as the mean \pm standard error of the mean of 6 rats per group $\left({ }^{\#} \mathrm{P}<0.05\right.$ vs. Con group; "P $<0.05$ vs. BPH group). BPH, benign prostatic hyperplasia; Fina, BPH-induced group treated with finasteride $5 \mathrm{mg} / \mathrm{kg} / \mathrm{day}$; MSJ, BPH-induced group treated with musulju $200 \mathrm{mg} / \mathrm{kg} / \mathrm{day}$; Con, control; iNOS, inducible nitric oxide synthase; COX-2, cyclooxygenase 2. 
A

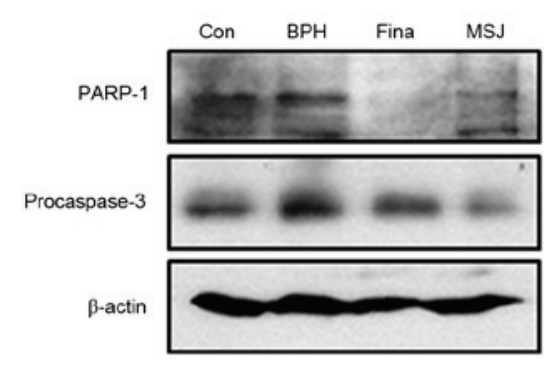

B

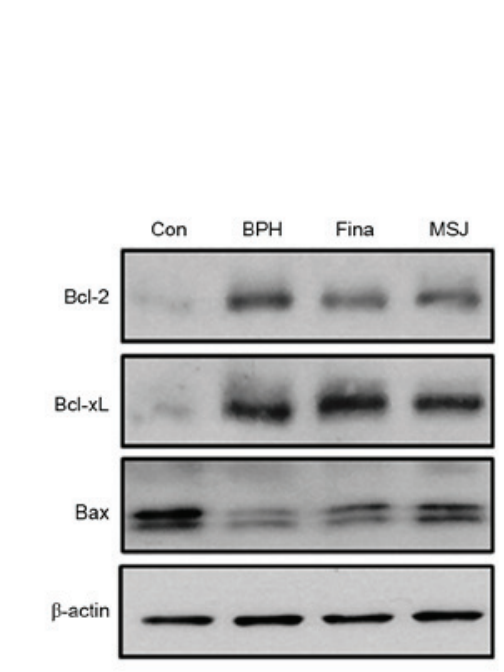

PARP-1
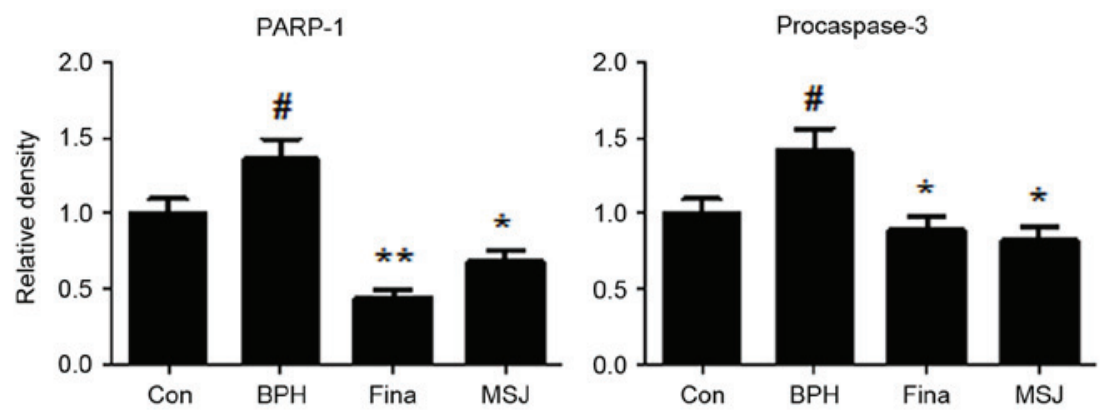
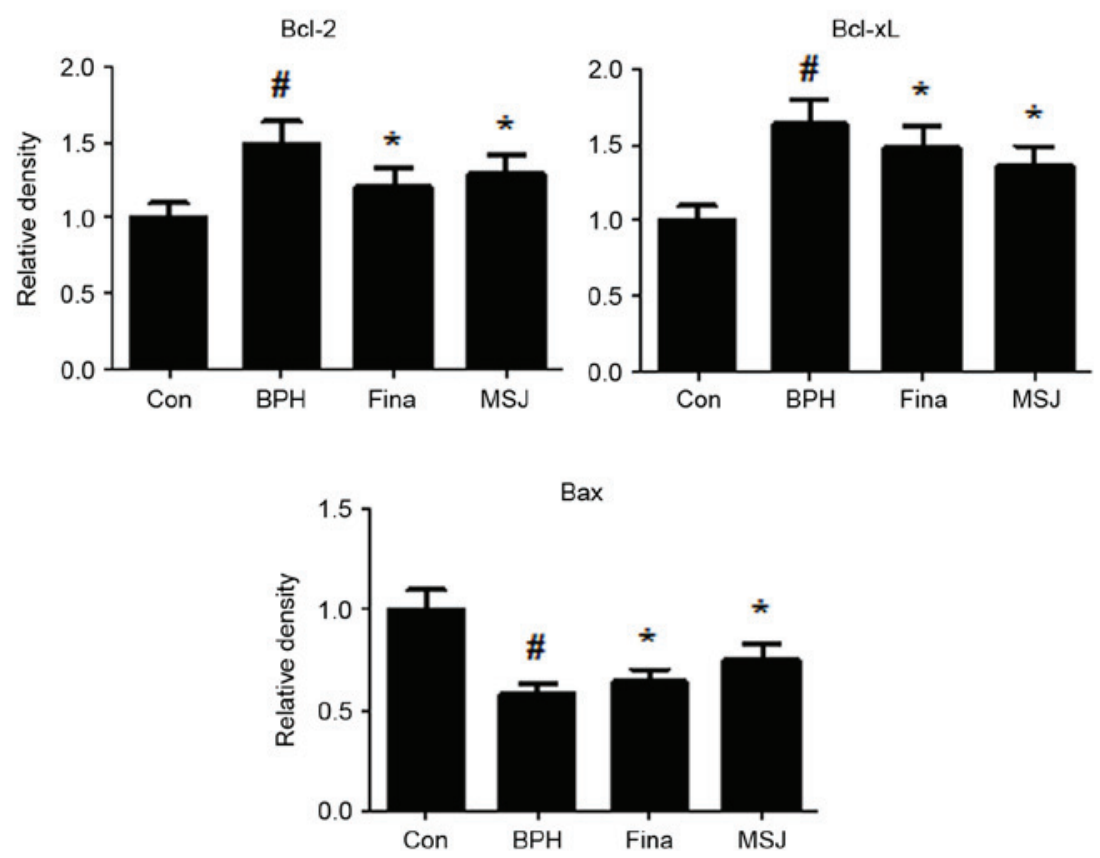

C

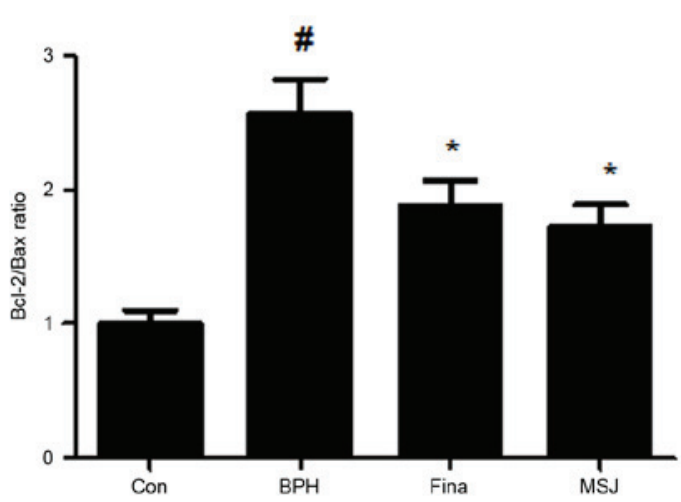

Figure 5. Effect of MSJ administration on the expression of apoptosis-related proteins in prostate tissues of BPH-induced rat models. (A) The expression levels of (A) PARP-1 and procaspase-3, and (B) Bcl-2 family were determined by western blotting using specific antibodies. $\beta$-actin was used as internal controls. The protein band densities were determine by densitometric analysis. (C) The densitometric analysis of Bcl-2 and Bax bands was performed, and the data were plotted as the $\mathrm{Bcl}-2 / \mathrm{Bax}$ ratio. Densitometric analysis was performed using Bio-rad Quantity One ${ }^{\circledR}$ Software. The data are presented as the mean \pm standard error of the mean of 6 rats per group $\left({ }^{*} \mathrm{P}<0.05\right.$ vs. Con group; ${ }^{*} \mathrm{P}<0.05,{ }^{* *} \mathrm{P}<0.01$ vs. $\mathrm{BPH}$ group). BPH, benign prostatic hyperplasia; Fina, BPH-induced group treated with finasteride $5 \mathrm{mg} / \mathrm{kg} / \mathrm{day}$; MSJ, BPH-induced group treated with musulju $200 \mathrm{mg} / \mathrm{kg} / \mathrm{day}$; Con, control; PARP, poly (ADP-ribose) polymerase-1; Bcl, B-cell lymphoma; Bax, Bcl-2-associated X protein.

Inflammation is extremely common in the prostates of aging men and is diagnosed histologically by the presence of inflammatory cells infiltrating the prostatic stroma, epithelium and/or ductal lumen (16). Prostatic inflammation involves both an inflammatory cell infiltrate and local tissue responses. A variety of inflammatory mediators, such as cytokines, chemokines, histamine, prostanoids, reactive oxygen species, growth factors, neuropeptides, and neurotrophins, may 
produce these effects (19). In the present study, MSJ markedly suppressed the expression of iNOS and COX-2, and these data suggested that MSJ may be a potent alternative medicine for the treatment of BPH via the regulation of the expression of inflammation-associated proteins.

Changes in the expression levels of Bcl-2 and caspase- 3 have been observed in BPH relative to healthy prostates. In normal prostatic tissue, Bcl-2 and caspase-3 expression in epithelial cells was either undetectable or only weakly detected. Therefore, it was suggested that lowering apoptosis rate in BPH may be associated with alterations in the balance between Bcl-2 and caspase-3 (20). Bcl-2 is an inner mitochondrial membrane protein, and its predominant effect is to prolong cell survival by inhibiting apoptosis. This increase in the expression of Bcl-2 may balance the death cascade by inhibiting the mitochondrial release of cytochrome $c$, which activates caspases. The caspase family of proteases is central to the cell death pathway. The extrinsic and intrinsic cell death pathways converge to activate caspase-3 for the final execution of apoptosis (21). Activation of caspase-3 cleaves a broad range of cellular substrates and promotes activation of a DNA endonuclease required for internucleosomal DNA fragmentation, a widely accepted hallmark of apoptosis. In the present study, it was revealed that MSJ activated effector caspase- 3 and decreased the protein expression of its substrate, PARP-1, in the $\mathrm{BPH}$ group. Additionally, MSJ markedly reduced the level of $\mathrm{Bcl}-2$ protein expression and increased the level of Bax protein expression in the rat BPH model. These data suggested that MSJ induced apoptosis through an antiapoptotic mechanism involving a decrease in the ratio of Bcl-2 to Bax, leading to a caspase-dependent pathway involving a caspase-3 activation.

In conclusion, the present study demonstrated that MSJ decreased prostate weight and testosterone production in BPH-induced rats. These effects may be due to the anti-inflammatory and apoptotic effects of MSJ. Accordingly, the use of MSJ as a potential therapeutic agent for the treatment of BPH must be further explored.

\section{References}

1. Roehrborn CG: Male lower urinary tract symptoms (LUTS) and benign prostatic hyperplasia (BPH). Med Clin North Am 95: 87-100, 2011.

2. Ho CK and Habib FK: Estrogen and androgen signaling in the pathogenesis of BPH. Nat Rev Urol 8: 29-41, 2011.
3. Huggins C and Hodges CV: Studies on prostatic cancer. I. The effect of castration, of estrogen and of androgen injection on serum phosphatases in metastatic carcinoma of the prostate. 1941. J Urol 168: 9-12, 2002.

4. Izumi K, Li L and Chang C: Androgen receptor and immune inflammation in benign prostatic hyperplasia and prostate cancer. Clin Investig (Lond) 4: 935-950, 2014.

5. Kramer G, Mitteregger D and Marberger M: Is benign prostatic hyperplasia $(\mathrm{BPH})$ an immune inflammatory disease? Eur Urol 51: 1202-1216, 2007.

6. Shoskes DA: Phytotherapy in chronic prostatitis. Urology 60 (Suppl 6): S35-S37; discussion 37, 2002.

7. Hirsch IH: Integrative urology: A spectrum of complementary and alternative therapy. Urology 56: 185-189, 2000.

8. Guo QL, Ding QL and Wu ZQ: Effect of baicalein on experimental prostatic hyperplasia in rats and mice. Biol Pharm Bull 27: 333-337, 2004.

9. Rick FG, Schally AV, Block NL, Nadji M, Szepeshazi K, Zarandi M, Vidaurre I, Perez R, Halmos G and Szalontay L: Antagonists of growth hormone-releasing hormone $(\mathrm{GHRH})$ reduce prostate size in experimental benign prostatic hyperplasia. Proc Natl Acad Sci USA 108: 3755-3760, 2011.

10. Maggi CA, Manzini S, Giuliani S and Meli A: Infravesical outflow obstruction in rats: A comparison of two models. Gen Pharmacol 20: 345-349, 1989.

11. Marinese D, Patel R and Walden PD: Mechanistic investigation of the adrenergic induction of ventral prostate hyperplasia in mice. Prostate 54: 230-237, 2003.

12. Miller J and Tarter TH: Combination therapy with dutasteride and tamsulosin for the treatment of symptomatic enlarged prostate. Clin Interv Aging 4: 251-258, 2009.

13. Wei JT, Calhoun E and Jacobsen SJ: Urologic diseases in America project: Benign prostatic hyperplasia. J Urol 173: 1256-1261, 2005.

14. Izumi K, Mizokami A, Lin WJ, Lai KP and Chang C: Androgen receptor roles in the development of benign prostate hyperplasia. Am J Pathol 182: 1942-1949, 2013.

15. Pawlicki B, Zielinski H and Dabrowski M: Role of apoptosis and chronic prostatitis in the pathogenesis of benign prostatic hyperplasia. Pol Merkur Lekarski 17: 307-310, 2004 (In Polish).

16. Corona G, Vignozzi L, Rastrelli G, Lotti F, Cipriani S and Maggi M: Benign prostatic hyperplasia: A new metabolic disease of the aging male and its correlation with sexual dysfunctions. Int J Endocrinol 2014: 329456, 2014.

17. Carson C III and Rittmaster R: The role of dihydrotestosterone in benign prostatic hyperplasia. Urology 61 (4 Suppl 1): S2-S7, 2003.

18. Steers WD: 5alpha-reductase activity in the prostate. Urology 58 (6 Suppl 1): S17-S24; discussion 24, 2001.

19. Meyer-Siegler KL and Vera PL: Substance P induced release of macrophage migration inhibitory factor from rat bladder epithelium. J Urol 171: 1698-1703, 2004.

20. Zhang X, Zhang Q, Zhang Z, Na Y and Guo Y: Apoptosis profiles in benign prostatic hyperplasia: Close associations of cell kinetics with percent area density of histologic composition. Urology 68: 905-910, 2006.

21. Nuñez G, Benedict MA, Hu Y and Inohara N: Caspases: The proteases of the apoptotic pathway. Oncogene 17: 3237-3245, 1998. 